This is a good little book. The views expressed are not necessarily traditional but are intended in places to provoke and stimulate investigation. It should certainly be read by postgraduate students interested in orthopaedics and there is much here for all, even the pre-clinical student.

\section{Mechanisms of Contraction of the Normal and Failing Heart}

By E. Braunwald, J. Ross and E. H. Sonnenblick. Pp. xi +205 , illustrated. New England Journal of Medicine, Progress Series. London: J. \& A. Churchill, 1968. £4.

Lewis called cardiac failure 'the central problem' of cardiology, and it remains so. For many years Eugene Braunwald and his colleagues have devoted their researches to increasing understanding of the mechanisms of cardiac muscle contraction. All over the world, appetites whetted by articles such as the recent Medical Progress Reports in the New England Journal of Medicine, cardiologists have been awaiting the publication of this monograph. It fully realizes their expectations.

Beginning with a completely up-to-date account of the ultramicroscopic structure of the sarcomere, and of the mechanical and biochemical events associated with its contraction, the authors demonstrate that the sarcomere lengthtension curve forms the ultrastructural basis of Starling's Law. The mechanics of the sarcomere leads on to an account of the mechanics of isolated heart muscle, which yields in turn to a consideration of the mechanisms of contraction of the intact ventricle. Changes in force-velocity relations in isolated muscle are shown to demonstrate the two general properties of heart muscle: a change in the initial muscle length - the basis of the Frank-Starling mechanism, and a change in the contractile state of the muscle, such as occurs through inotropic intervention. The mechanics of contraction of the intact ventricle are much more complicated than even recent simple modifications of the Frank-Starling curves would suggest. The authors demonstrate that a useful framework for consideration of the behaviour of the intact heart is a three dimensional diagram relating velocity of contraction, length of muscle, and load. The efficiency of the heart is related not only to the mechanics of contraction, but also to energetics, so the metabolic aspects of 'mechanochemical coupling' in heart muscle are described.

From the heart of the experimental animal the authors turn to the human heart. The role of the autonomic system, exercise and drugs in cardiac behaviour are well described, both for the normal and for the failing heart. The stroke volume of the intact ventricle is determined by three influences: the end-diastolic muscle length, the tension the muscle develops during contraction, and the contractile state of the muscle.

The final chapter of the book synthesizes all the previous arguments, and demonstrates by means of a family of Frank-Starling curves that the stretching of the myocardium and its contractile state both influence ventricular performance at various levels of bodily activity, in the normal and in the failing heart.

This is an extremely important book which will become a classic. It is wonderful that in this age of medical bibliomegaly the authors have managed to say so much of such great value, including over 250 references, in less than 200 pages. It only just fails to fit into a jacket pocket. This admirable size has been achieved by compact and economical writing. There is not one sentence of padding. The logical steps in the argument are closely reasoned and succinctly stated. The many diagrams, which are complicated but clear, are an essential feature of the book, so that text and diagrams are complementary.

It is not a book for the skimmer. The concentration of facts, ideas and arguments demands an equal concentration by the reader, but he will find that the effort is fully rewarded.
The price may seem high for such a small volume, but in terms of much present intellectual currency each page contains more than fivepence worth of ideas. All cardiologists and physiologists should read this book.

\section{Progress in Gastroenterology. Vol.}

Edited by George B. Jerzy Glass. Pp. 520, illustrated. London and New York: Grune \& Stratton, 1968. \$29.50.

The modern gastroenterologist must be kept informed about the wealth of information yielded by current physiological, biochemical, enzymological and immunologic research and Dr Jerzy Glass in this excellent volume on progress in gastroenterology, has succeeded in doing just this. He has not attempted a complete coverage of the subject but has selected twenty topics, covered by thirty-two authors, who have built up international reputations on their particular interests and who were asked to express their concepts freely without fear of editorial restraint. The research reported is indeed world-wide, authors being asked to contribute from America, Australia, England, France, Spain and South Africa.

Chapters will be found to interest the many disciplines concerned with the practice of gastroenterology. For those clinically orientated, two chapters are contributed on gastritis by Taylor \& Fisher and by Katz \& Siegal, single chapters on cardiorespiratory abnormalities after gastric haemorrhage in cirrhotics by Louis Del Guercio, on medium chain tryglycerides by Peter Holt, and on the pathophysiology and management of extensive small bowel resections by Winawer and Zamchek. Two chapters are of particular interest to all physicians. These are by Dr Trevor Cooke, who summarizes 25 years' experience in the diagnosis and management of adult coeliac disease, and Dr Marks, Dr Bank and Dr Louw, who describe their vast experience in the diagnosis and management of pancreatitis in their South African population.

For the gastric physiologist the use of the rat as an experimental animal to investigate ulcer and post-gastrectomy problems is described by Rene Lambert from Lyon, and the evidence for an endogenous secretory inhibitor, gastrone, is reviewed by Dr Jerzy Glass and Dr Charles Code. The proteolytic enzymes and the organic constituents of gastric juice are described by Tang and Stewart Wolf and by Piper, the latter author describing their value in the diagnosis of gastric carcinoma. The properties of the intrinsic factor and allied proteins which facilitate $\mathbf{B}_{12}$ transport are described by Kai Simons. The physiology of fat absorption in normal and pathological states and the functional correlation with villous form and motility are described and are beautifully illustrated by electron microscope studies by the North Carolina team of Sessions, Viegas de Androde, Kokas and Dobbins.

Of particular value to digestive endoscopists is a chapter by Henry Colcher who contrasts and compares the various types of gastroscope, fibrescope and gastrocamera now available and details a vast experience of gastrophotography and cinegastroscopy. The pathologist will be interested in a re-evaluation of gastric cytology in gastric carcinoma from Spain by Dr Vilardell and the immunologist in a review of the immune mechanisms concerned in the pathogenesis of ulcerative colitis by $\mathrm{Dr}$ Watson and $\mathrm{Dr}$ Bolt from Ann Arbor. Finally, the needs of the radiologist are catered for by two profusely illustrated chapters on granulomatous colitis by Marshak \& Lindner and on angiography in digestive diseases by Sybers \& Galambos.

The book is beautifully printed, well illustrated and bound and for this reason justifies its rather high price. It can be strongly recommended to all physicians, surgeons and postgraduate students interested in gastroenterology while special chapters are of interest to general physicians, pathologists and radiologists. 